\title{
Self-Assembled Three Dimensional Radio Frequency (RF) Shielded Containers for Cell Encapsulation
}

\author{
Barjor Gimi, ${ }^{1}$ Timothy Leong, ${ }^{2}$ Zhiyong $\mathrm{Gu},{ }^{2}$ Michael \\ Yang, ${ }^{2}$ Dmitri Artemov, ${ }^{1}$ Zaver M. Bhujwalla, ${ }^{1}$ and \\ David H. Gracias ${ }^{2,3, *}$ \\ ${ }^{1}$ The Russell H. Morgan Department of Radiology and Radiological \\ Sciences, The Johns Hopkins University School of Medicine, Balti- \\ more, MD 21205 \\ ${ }^{2}$ Department of Chemical and Biomolecular Engineering, $3400 \mathrm{~N}$. \\ Charles Street, 125 MD Hall, Baltimore, MD 21218 \\ ${ }^{3}$ Department of Chemistry, Johns Hopkins University, Baltimore, MD \\ 21218, USA \\ E-mail: dgracias@jhu.edu
}

\begin{abstract}
This paper describes the construction of three dimensional (3D) encapsulation devices in large numbers, using a novel selfassembling strategy characterized by high mechanical stability, controlled porosity, extreme miniaturization, high reproducibility and the possibility of integrating sensing and actuating electromechanical modules. We demonstrated encapsulation of microbeads and cells within the containers, thereby demonstrating one possible application in cell encapsulation therapy. Magnetic resonance (MR) images of the containers in fluidic media suggest radio frequency (RF) shielding and a susceptibility effect, providing characteristic hypointensity within the container, thereby allowing the containers to be easily detected. This demonstration is the first step toward the design of 3D, micropatterned, non-invasively trackable, encapsulation devices.
\end{abstract}

Key Words. 3D, microtechnology, self assembly, cell encapsulation, MRI

\section{Introduction}

In recent years, advances in regenerative medicine have inspired therapies targeted at the cellular level. For example, a wide range of cell lines have been enclosed within semi permeable and biocompatible immobilization devices that control cell release and the bidirectional diffusion of molecules (Lanza et al., 1996; Orive et al., 2002). Transplanted cells have been used to secrete hormones (O'Shea and Sun, 1986), neurotransmitters (Aebischer et al., 1991), growth/inhibition factors (Sagot et al., 1995) and for gene therapy (Pizzorusso et al., 1997). Cellular transplantation has also been suggested as a way to overcome acute human organ shortage. Concurrent advances in microtechnology have impacted medicine, as new implantable devices, microarrays, nanoporous biocapsules and microprobes are developed. In addition to cellular encapsulation, these devices have facilitated on-demand drug release and early diagnosis of diseases (Desai et al., 1997; Santini et al., 1999; Kost and Langer, 2001; Leoni and Desai, 2004; Lesinski et al., 2005). In contrast to poly- meric, hydrogel and sol-gel based processes that have been used for encapsulation and delivery (Lim and Sun, 1980; Chang, 2005), conventional silicon ( $\mathrm{Si}$ ) based microfabrication has high reproducibility and stability. Si based devices also have the capacity to integrate electronic and optical modules that can be used for sensing, tracking and actuation. However Si based microfabrication is principally a two dimensional (2D) process (Madou, 2002), hence three dimensional (3D) micropatterned devices remain largely unexplored. A 3D micropatterned device has several advantages over its $2 \mathrm{D}$ counterpart-a larger surface area to volume ratio, thereby maximizing interactions with the surrounding medium and providing space to mount different electromechanical modules, and a finite volume allowing encapsulation of functional elements. We report on a strategy to construct 3D micropatterned devices for cellular encapsulation, using self-assembly.

\section{Design of the 3D Micropatterned Encap- sulation Devices}

The 3D encapsulation devices described here are hollow containers with linear dimensions of $200 \mu \mathrm{m}$. As compared to smaller or larger sized devices, the $200 \mu \mathrm{m}$ size provides the maximum encapsulation volume while still allowing the diffusion of oxygen and nutrients to the cells. It is known that if cells are more than 150-200 $\mu$ m away from the nearest blood vessel, the environment becomes hypoxic (Thomlinson and Gray, 1955). In principle, the fabrication strategy would work on smaller or larger size scales in the design of containers for other applications.

The containers were designed as copper $(\mathrm{Cu})$ based microelectronic devices that function as radio frequency

\footnotetext{
*Corresponding author.
} 
(RF) shields to facilitate their detection using magnetic resonance imaging (MRI). The surfaces of the containers were patterned with microscale perforations to demonstrate controlled porosity. The dimension of the pores were orders of magnitude smaller than the wavelength of the oscillating magnetic field in the MRI scanner operating at $500 \mathrm{MHz}$ and hence had no adverse effect on the shielding. The thickness of the surfaces of the container were designed to be larger than the conductor skin depth $(\delta)$ at the frequency of the radiation (Tsaliovich, 1999), resulting in low conductor resistance and ensuring that eddy currents persist long enough to maintain shielding during the time of image acquisition. The skin depth of $\mathrm{Cu}$ at $500 \mathrm{MHz}$ is $2.9 \mu \mathrm{m}$ (Kittel, 1996), hence, we designed containers to have frames with thickness ranging from $7-15 \mu \mathrm{m}$.

In addition to the diamagnetic $\mathrm{Cu}$ containers, we also fabricated ferromagnetic nickel (Ni) containers to investigate the effect of magnetic susceptibility on the MR im-

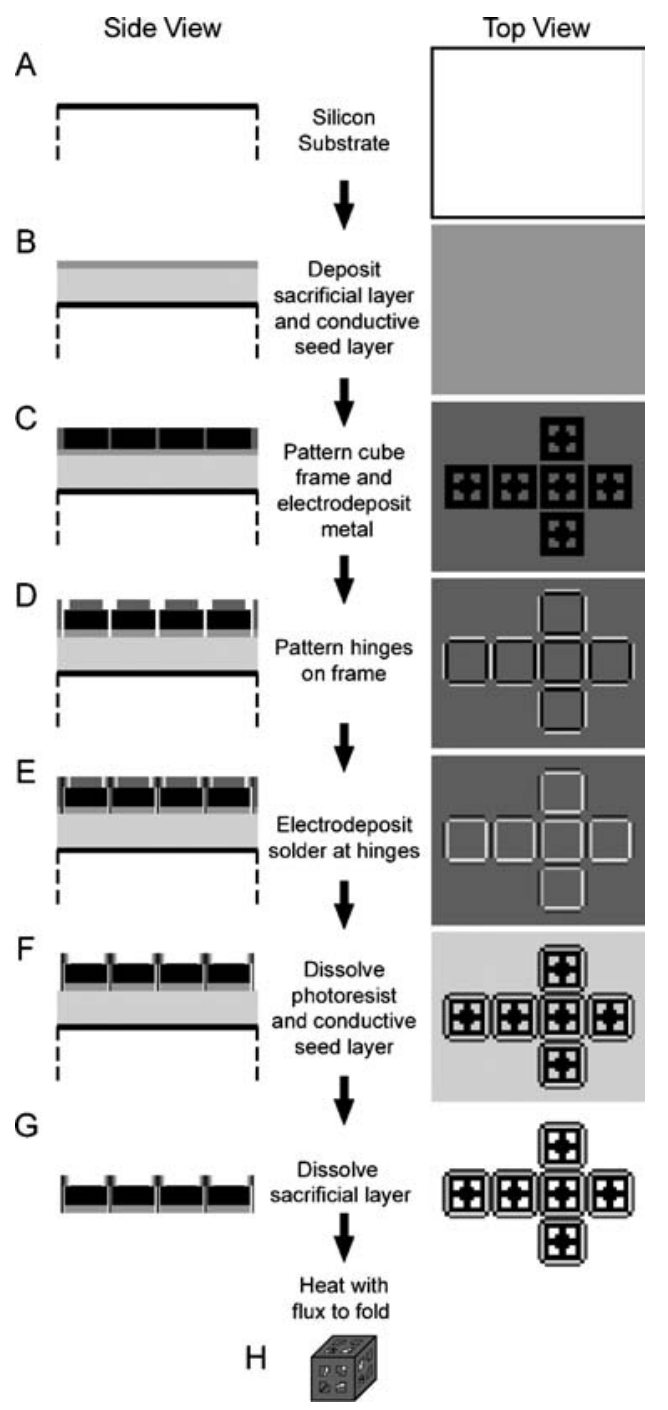

ages of the container. Magnetic field distortions resulting from the differences in magnetic susceptibility between an object and its surrounding medium cause a loss of phase coherence in the magnetization of the sample. Since the magnetic susceptibility of $\mathrm{Cu}$ is comparable to that of water, while that of $\mathrm{Ni}$ is orders of magnitude higher than that of water, a more pronounced distortion was expected for $\mathrm{Ni}$ containers in aqueous media (Bartels et al., 2001).

\section{Microfabrication and Self-Assembly}

We used microfabrication and surface tension driven selfassembly (Terfort et al., 1997; Harsh et al., 1999; Hui et al., 2000; Gracias et al., 2002) to fabricate and fold a $2 \mathrm{D}$ precursor into a $3 \mathrm{D}$ hollow structure. The fabrication process involved three steps: (1) patterning the surfaces on the $2 \mathrm{D}$ precursor (2) patterning hinges between the surfaces, and (3) self-assembly of the 2D precursor (Figure 1). It is possible to pattern the surfaces of the precursor using most microfabrication processes including lithography, thin film deposition, etching and ion implantation.

Fig. 1. The process flow used to fabricate the containers. $(A-B) A$ $15 \mathrm{~nm}$ layer of $\mathrm{Cr}$ followed by a $100 \mathrm{~nm}$ thick layer of $\mathrm{Cu}$ were evaporated on top of a $5 \mu \mathrm{m}$ thick sacrificial layer of polymethyl methacrylate (PMMA) that was spun on a Si wafer. The Cr layer functions as an adhesive promoter while the Cu layer functions as a conductive seed layer for subsequent electrodeposition. (C) After thin film deposition, the substrate was patterned using photolithography on the photoresist Shipley SPR220 (Rohm and Haas, www.rohmhaas.com). The photomask used to pattern the resist was a transparency mask with six $200 \mu \mathrm{m}$ squares spaced $20 \mu \mathrm{m}$ apart. After photolithography, electrodeposition was used to build pattern the metallic surfaces of the container in the photoresist mold up to a height of 7-15 $\mu \mathrm{m}$, using commercial electrolytic solutions (Technic, Inc, www.technic.com) containing the metal ions of choice ( $\mathrm{Cu}$ or $\mathrm{Ni}$ and $\mathrm{Au}$ ). The Au was used to protect the Cu surface from subsequent etching steps and render it inert. (D) A second photolithography step was used to align the hinges to the square faces. The hinge mask consisted of wider hinges $\left(50 \times 160 \mathrm{\mu m}^{2}\right)$ at the interfaces of adjacent squares and narrower hinges $\left(25 \times 160 \mathrm{\mu m}^{2}\right)$ at the edges of the squares. After photolithography and prior to hinge electrodeposition, the exposed $\mathrm{Cu}$ and $\mathrm{Cr}$ in the area of the hinges were etched using commercial etchants (Technic, Inc, www.technic.com). (E) Pure tin (m.p. $232^{\circ}$ C) or tin/lead $\left(\mathrm{Sn} / \mathrm{Pb}:\right.$ m.p. $\left.183^{\circ} \mathrm{C}\right)$ solder was then electroplated in the hinge regions. $(F-G)$ After hinge deposition, the seed layers were etched and the $2 D$ precursor precursors were released from the wafer on immersion in a solution of N-Methyl Pyrrolidone. (H) Approximately 50 precursors were then scattered in a small crystallizing dish using a pipette. A very thin layer of \#5RMA flux, (Indium Corporation, www.indium.com, to dissolve any oxide formed on the solder) was poured into the dish. The dish was then heated to $100^{\circ} \mathrm{C}$ for a $2-3 \mathrm{~min}$ and then ramped up to $250^{\circ} \mathrm{C}-300^{\circ} \mathrm{C}$ for $20 \mathrm{sec}$. Because of the low volume of flux, the agitation was sufficient to correct for defects in the folding but not large enough to cause the precursors to collide into each other and become fused. The molten solder generated the force to fold the $2 D$ precursors into $3 D$ containers. On cooling, the containers were permanently held together by solid solder hinges. 


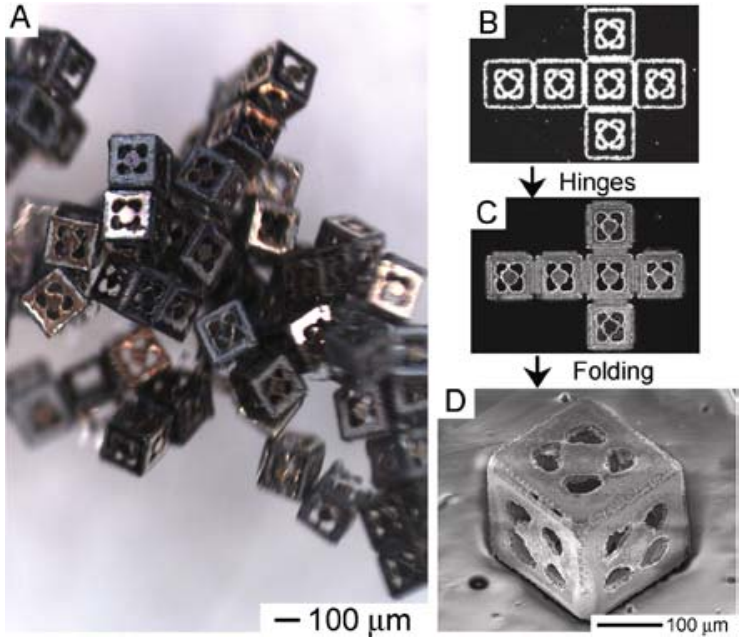

Fig. 2. (A) Optical image showing a collection of biocontainers. (B-D) Optical and Scanning electron microscopy (SEM) images at different stages of the fabrication process: $(B)$ the $2 D$ precursor with electrodeposited surfaces, $(C)$ the precursor with surfaces and hinges, and $(D)$ the self-assembled biocontainer.

We used thin film deposition, photolithography and electrodeposition on a sacrificial layer to pattern single and multiple perforations on the surfaces of the precursor. In a second layer of photoresist, hinges were patterned on the edges of the surfaces. The hinge width between adjacent surfaces was twice the hinge width at the edges so that all hinged joints had equal solder volume upon self-assembly. The solder volume was critical to ensure a folding angle of $90^{\circ}$ (Syms et al., 2003). The 2D precursors were then lifted off the wafer by dissolution of the sacrificial layer. The containers self-assembled when the precursors were heated above the melting point of the solder, wherein the liquid solder with high surface tension generated the force required to assemble adjacent surfaces. The fabrication strategy allows a large number of devices to be constructed in a single process run (Figure 2(A)). Figure 2 (B)-(D) shows optical and SEM images of the micropatterned containers at different stages of the process: the 2D precursor with electrodeposited surfaces, the precursor with surfaces and hinges, and the self-assembled container.

\section{Cell Encapsulation}

In order to demonstrate encapsulation, open faced cubic containers (Figure 3(A)) were used solely for easy visualization of the contents during encapsulation and release. For other applications, it may be desirable to use this strategy to construct containers with selectively sealed or micro/nano perforated surfaces, and fabricate more complex, polyhedral structures with rounded vertices. We loaded containers with microbeads (Figure 3(B)) since many cellular delivery techniques employ microbeads with cells adhered to their surface. A suspension of the beads in ethanol was pipetted onto the container. When the ethanol evaporated, the beads were held together by van der Waals forces. The glass beads could be released by pulsatile agitation of the container. MDA-MB-231 (Cailleau et al., 1974) breast cancer cells, in an extracellular matrix (ECM) suspension, were also loaded into a container. The cancer cells are representative of rapidly proliferating cells and immortalized cells such as $\beta$ TC 3 cells used in diabetes therapy, and stem cells used in regeneration. First, a suspension of $5 \%$ agarose gel was micropipetted (60 $\mu \mathrm{m}$ tip) into the container using a stereotactic manipulator. The gel adhered to the sides of the container thereby sealing the surfaces and leaving a void in the center. The cell-ECM suspension at $4{ }^{\circ} \mathrm{C}$ was then microinjected into this void, and subsequently gelled at $37^{\circ} \mathrm{C}$. The cells were stained with the fluorescent dye, Calcein-AM (Sigma-Aldrich), (Figure 3(C)), which stains positively for live cells. The cells were viable within the container even in the absence of cell culture medium and could be released by immersion and agitation of the container in a warm cell culture medium (Figure 3(D)). We envision that such cellular encapsulation and release can be used in regenerative medicine and in placing inocula in animal models for cancer. If the microcontainers are used in vivo, special care will be necessary to ensure their biocompatibility. Apart from any issues with toxicity, biofouling and the formation of fibrotic tissue around the implant can limit their utility in the body (Rihova, 2000; Walczak et al., 2005). The containers used in this demonstration had a thin gold coating on the interior surfaces to provide bio-inertness. The biocompatibility of the micontainers can be further enhanced by precisely controlling the materials used, surface chemistry and shape. One such approach would be to coat the entire self-assembled 3D microcontainer with a layer of an inert metal (by electrodeposition), oxide (by chemical vapour deposition) or a polymer (by immersion or vapor coating) (Rihova, 2000; Johnston et al., 2005). The surface of noble metals can also be readily modified using a variety of self-assembled organic monolayers that are designed to reduce non-specific adsorption of proteins and subsequent biofilm formation (Ostuni et al., 2001).

\section{MR Detection and Tracking}

We demonstrated non-invasive detection of the containers by embedding them in $5 \%$ agarose gel and imaging them with MRI in a $500 \mathrm{MHz}$ vertical bore Bruker Avance microimaging system. Figure 4 shows MR images of a 

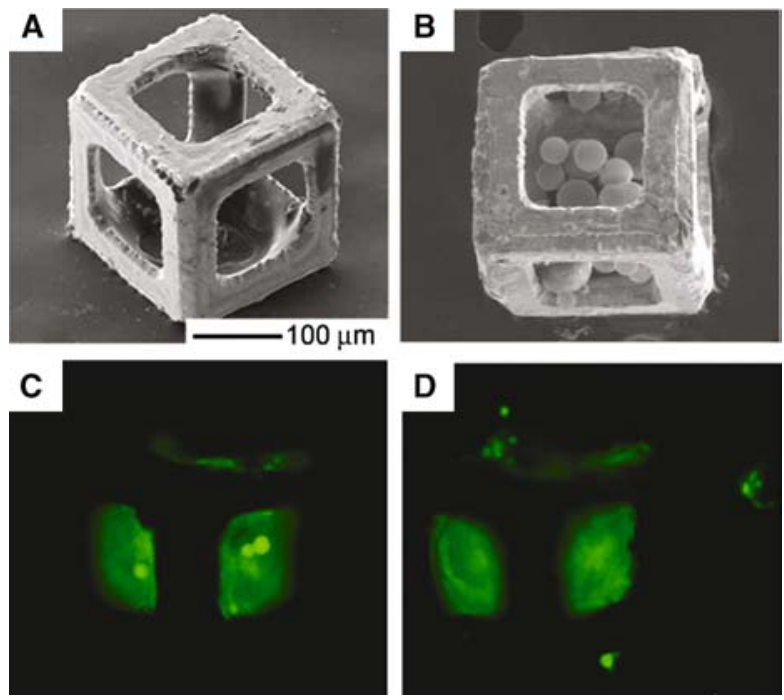

Fig. 3. (A) SEM image of (A) a hollow, open surfaced, biocontainer, and $(B)$ a device loaded with glass microbeads. (C) Fluorescence microscopy images of a biocontainer loaded with cell-ECM-agarose with the cell viability stain, Calcein-AM. (D) Release of viable cells from the biocontainer.

$900 \mu \mathrm{m}$ diameter capillary containing a $\mathrm{Cu}$ (Figure 4(A)) and a Ni (Figure 4(B)) container embedded in agarose gel. For the images shown here, we used a 3D FLASH sequence with the echo time (TE) in the range of 4$6 \mathrm{~ms}$, a repetition time (TR) of $50 \mathrm{~ms}$, flip-angle of $30^{\circ}$, and a spatial resolution of $25 \mu \mathrm{m} \times 25 \mu \mathrm{m} \times 20 \mu \mathrm{m}$. We observe a characteristic signature for both the $\mathrm{Cu}$ and the $\mathrm{Ni}$ containers - there is a pronounced darkness in the region of each container. The hypointense signatures have been observed before in MRI of larger centimeter scale metallic coils (Shenhav and Azhari, 2004). While the region of hypointensity in the MR image was comparable to the size of the non-magnetic $\mathrm{Cu}$ container, it was much larger for the ferromagnetic $\mathrm{Ni}$ container due to a pronounced susceptibility effect (Schueler et al.,
1999). We observe a characteristic hypointense signature irrespective of the pattern on the surfaces of the container.

To demonstrate RF shielding in the $\mathrm{Cu}$ containers, the near magnetic field response in the vicinity of the container was simulated using a finite element electromagnetic simulation package, FEKO (EM Software \& Systems-SA Ltd, www.feko.info/). A full-wave method of moments approach was used to simulate the near magnetic field in the region of a $200 \mu \mathrm{m}$ wire frame with wire segments of $8 \mu \mathrm{m}$ radius, assuming perfect electric conductors coated with copper (conductivity $=5.813 \times 10^{7} \mathrm{~S} \cdot \mathrm{m}^{-1}$ ). The simulation of the cubical wire frame model was performed with a linear polarized plane wave excitation at $500 \mathrm{MHz}$; we used an excitation source of $1 \mathrm{~V} / \mathrm{m}$ incident on the wire frame, with $\mathbf{E}$ in the $z$ direction and $\mathbf{H}$ in the $y$ direction. The copper wire frame was assigned a relative permeability of 1 , thereby simulating only the RF shielding effect and not the susceptibility effects. Figure 4(C)-(D) shows the near magnetic field response in both the $x-y$ and the $y-z$ central planes.

We tracked the containers spatio-temporally in flow through an S-shaped $500 \mu \mathrm{m}$ diameter fluidic channel. The channel was fabricated by molding poly dimethyl siloxane (PDMS) in an SU-8 photoresist mold that was patterned using photolithography. The channel was sealed with a second, flat, oxygen plasma treated PDMS layer. Polyethylene tubes were connected to the inlet and outlet ports of the channel, the channel was flushed with silicone oil and the container was introduced into the channel. Under pressure driven flow, the container progressed through the channel and was imaged at different positions (Figure 5). The sequence of MR images were acquired with a 2D RARE sequence with TE $=42 \mathrm{~ms}, \mathrm{TR}=1077 \mathrm{~ms}$, and a spatial resolution of $50 \mu \mathrm{m} \times 50 \mu \mathrm{m} \times 500 \mu \mathrm{m}$. This ready trackability with MRI, at short echo times, without the need for a contrast agent, highlights a major advantage of the encapsulation device as compared to existing systems.
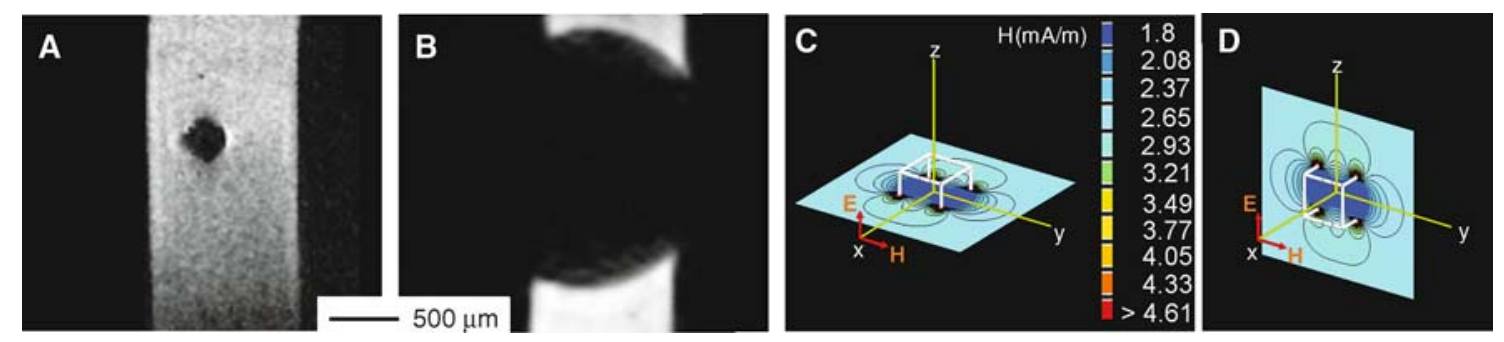

Fig. 4. MR images of an open faced (A) non-magnetic Cu container and (B) ferromagnetic Ni container. (C-D) Finite element simulation results of the near magnetic field in the region of a Cu container, in the $(C) x y$ and $(D) y z$ central planes. The magnetic field distortions and the shielding effect caused by the wire frame are evident. 


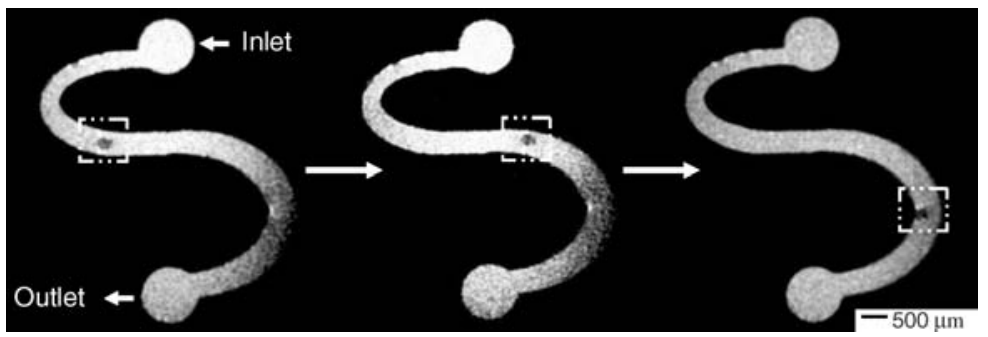

Fig. 5. MR tracking of a container in a fluidic channel. MR images of the container at different time points taken under pressure driven flow of the fluid.

\section{Concluding Remarks}

In conclusion, we have demonstrated a strategy that can be used to fabricate 3D, arbitrarily micropatternable, noninvasively trackable containers that allow selective perfusion between its content and the surrounding medium. The perforations on the surfaces of our devices are microsized, due to the fact that we have used microlithography to pattern the $2 \mathrm{D}$ templates. If nanoscale patterning techniques are used instead to pattern the $2 \mathrm{D}$ templates, it will be possible to pattern nanoporous faces, thereby generating $3 \mathrm{D}$ nanoporous containers that will allow highly selective bidirectional diffusion of molecules. We envision the use of several nanoscale patterning techniques including conventional nano lithography (Wallraff and Hinsberg, 1999) and novel methods that include selective etching, electrochemical etching and soft-lithography (Sinha et al., 2004; Gates et al., 2005).

Since the fabrication strategy described here is compatible with conventional 2D microfabrication, it may be possible to add electromechanical modules for remote activation, wireless communication, signal processing, and biosensing to the surfaces of the containers to enable medical diagnostics and therapeutics. We also envision that these 3D containers that function as small Faraday cages will find utility in other applications requiring electromagnetic shielding in small volumes.

\section{Acknowledgments}

This research was supported in part by the National Institutes of Health (NIH P50 CA 103175). We thank Dr. Richard L. Magin for useful discussions and Dr. Piotr Walczak for help with loading the containers with cells.

\section{References}

P. Aebischer, P.A. Tresco, S.R. Winn, L.A. Greene, and C.B. Jaeger, Exp. Neurol. 111, 269 (1991).

L.W. Bartels, H.F. Smits, C.J. Bakker, and M.A. Viergever, J. Vasc. Interv. Radiol. 12, 365 (2001)
R. Cailleau, R. Young, M. Olive, and W.J. Reeves, Jr., J. Natl. Cancer Inst. 53, 661 (1974).

T.M.S. Chang, Nat. Rev. Drug Discov. 4, 221 (2005).

T.A. Desai, W.H. Chu, J.K. Tu, G.M. Beattie, A. Hayek, and M. Ferrari, Biotechnol. Bioeng. 57, 118 (1997).

B.D. Gates, Q. Xu, M. Stewart, D. Ryan, C.G. Willson, and G.M. Whitesides, Chem. Rev. 105, 1171 (2005).

D.H. Gracias, V. Kavthekar, J.C. Love, K.E. Paul, and G.M. Whitesides, Adv. Mater. 14, 235 (2002).

K.F. Harsh, V.M. Bright, and Y.C. Lee, Sens. Actuators A 77, 237 (1999). E.E. Hui, R.T. Howe, and M.S. Rodgers, IEEE 13th Int. Conf. Microelectromech. Syst., 602 (2000).

E.E. Johnston, J.D. Bryers, and B.D. Ratner, Langmuir 2005, 870 (2005).

C. Kittel, Introduction to Solid State Physics (Wiley, New York, 1996).

J. Kost and R. Langer, Adv. Drug Deliv. Rev. 46, 125 (2001).

R.P. Lanza, J.L. Hayes, and W.L. Chick, Nat. Biotech. 14, 1107 (1996).

L. Leoni and T.A. Desai, Adv. Drug Deliv. Rev. 56, 211 (2004).

G. Lesinski, S. Sharma, K. Varker, P. Sinha, M. Ferrari, and W. Carson, Biomed. Microdevices 7, 71 (2005).

F. Lim and A.M. Sun, Science 210, 908 (1980).

M.J. Madou, Fundamentals of Microfabrication. 2nd ed (CRC Press, Boca Raton, Fla., 2002).

G. Orive, R.M. Hernandez, A.R. Gascon, M. Igartua, and J.L. Pedraz, Trends Biotech. 20, 382 (2002).

G.M. O'Shea and A.M. Sun, Diabetes 35, 943 (1986).

E. Ostuni, R.G. Chapman, M.N. Liang, G. Meluleni, G. Pier, D.E. Ingber, and G.M. Whitesides, Langmuir 17, 6336 (2001).

T. Pizzorusso, V. Porciatti, J.L. Tseng, P. Aebischer, and L. Maffei, Neuroscience 80, 307 (1997).

B. Rihova, Adv. Drug Deliv. Rev. 42, 65 (2000).

Y. Sagot, S.A. Tan, E. Baetge, H. Schmalbruch, A.C. Kato, and P. Aebischer, Eur. J. Neurosci. 7, 1313 (1995).

J.T. Santini, M.J. Cima, and R. Langer, Nature 97, 335 (1999).

B.A. Schueler, T.B. Parrish, J.C. Lin, B.E. Hammer, B.J. Pangrle, E.R. Ritenour, J. Kucharczyk, and C.L. Truwit, J. Magn. Reson. Imaging 9, 596 (1999).

A. Shenhav and H. Azhari, Magn. Reson. Med. 52, 1465 (2004).

P. Sinha, G. Valco, S. Sharma, X. Liu, and M. Ferrari, Nanotechnology 15, 585 (2004).

R.R.A. Syms, E.M. Yeatman, V.M. Bright, and G.M. Whitesides, J. Microelectromech. Syst. 387 (2003).

A. Terfort, N. Bowden, and G.M. Whitesides, Nature 86, 162 (1997).

R.H. Thomlinson and L.H. Gray, Br. J. Cancer 9, 539 (1955).

A.B. Tsaliovich, Electromagnetic Shielding Handbook for Wired and Wireless EMC Applications (Kluwer Academic, Boston, 1999).

R.J. Walczak, A. Boiarski, M. Cohen, T. West, K. Melnik, J. Shapiro, S. Sharma, and M. Ferrari, NanoBiotech. 1, 35 (2005).

G.M. Wallraff and W.D. Hinsberg, Chem. Rev. 99, 1801 (1999). 